\title{
Selective and nonselective inhibition of competitors in picture naming
}

\author{
Zeshu Shao • Antje S. Meyer • Ardi Roelofs
}

Published online: 29 May 2013

(C) Psychonomic Society, Inc. 2013

\begin{abstract}
The present study examined the relation between nonselective inhibition and selective inhibition in picture naming performance. Nonselective inhibition refers to the ability to suppress any unwanted response, whereas selective inhibition refers to the ability to suppress specific competing responses. The degree of competition in picture naming was manipulated by presenting targets along with distractor words that could be semantically related (e.g., a picture of a dog combined with the word cat) or unrelated (tree) to the picture name. The mean naming response time (RT) was longer in the related than in the unrelated condition, reflecting semantic interference. Delta plot analyses showed that participants with small mean semantic interference effects employed selective inhibition more effectively than did participants with larger semantic interference effects. The participants were also tested on the stop-signal task, which taps nonselective inhibition. Their performance on this task was correlated with their mean naming RT but, importantly, not with the selective inhibition indexed by the delta plot analyses and the magnitude of the semantic interference effect. These results indicate that nonselective inhibition ability and selective inhibition of competitors in picture naming are separable to some extent.
\end{abstract}

Keywords Delta plot $\cdot$ Lexical access $\cdot$ Nonselective inhibition $\cdot$ Picture-word interference $\cdot$ Selective inhibition

A key component of the human ability to speak is the retrieval of words from the mental lexicon. This process, called lexical

Z. Shao $(\bowtie) \cdot$ A. S. Meyer

Max Planck Institute for Psycholinguistics, P. O. Box 310,

$6500 \mathrm{AH}$, Nijmegen, the Netherlands

e-mail: Zeshu.Shao@mpi.nl

A. S. Meyer · A. Roelofs

Donders Institute for Brain, Cognition and Behaviour,

Radboud University, Nijmegen, the Netherlands access, has been widely studied using a range of different paradigms, including analyses of speech errors in healthy and brain-damaged speakers (e.g., Badecker, Miozzo, \& Zanuttini, 1995; Dell, Schwartz, Martin, Saffran, \& Gagnon, 1997; Goodglass, Kaplan, Weintraub, \& Ackerman, 1976; Kay \& Ellis, 1987), chronometric experiments (e.g., Schriefers, Meyer, \& Levelt, 1990), brain-imaging studies (e.g., de Zubicaray, Wilson, McMahon, \& Muthiah, 2001; Indefrey \& Levelt, 2004), and computational modeling (e.g., Foygel \& Dell, 2000). This research effort has led to the development of detailed models of lexical access (e.g., Caramazza, 1997; Dell, 1986; Levelt, 2001; Levelt, Roelofs, \& Meyer, 1999; Rapp \& Goldrick, 2000; Roelofs, 1992). Although differing in important ways, most models agree that lexical access to a word proceeds in two steps, the retrieval of a syntactic representation of the word (often called the lemma) and encoding of the corresponding morphological, phonological, and phonetic representations.

Speaking is a goal-directed activity. Speakers do not emit random words at random times but select words to achieve communicative goals. Thus, executive control must be involved in this process. Although there are a variety of conceptions of executive control processes (e.g., Baddeley, 1986; Posner \& Petersen, 1990), they all agree that one important component of executive control is the ability to inhibit competing information (Miyake et al., 2000). During speaking, many thoughts may come to mind that are not to be expressed, and many words may be activated that are not included in the utterance because, for instance, they are in a language not shared by the interlocutor or because they are too general or socially inappropriate. Intuition suggests that speakers need to inhibit such concepts and words. A number of recent empirical studies have suggested the involvement of inhibition in lexical access in monolingual and bilingual spoken word production (e.g., de Zubicaray, McMahon, Eastburn, \& Pringle, 2006; de Zubicaray, McMahon, Eastburn, \& Wilson, 2002; de Zubicaray et al., 2001; Guo, 
Liu, Misra, \& Kroll, 2011; Jackson, Swainson, Cunnington, \& Jackson, 2001; Misra, Guo, Bobb, \& Kroll, 2012; Roelofs, Piai, \& Garrido Rodriguez, 2011). Moreover, there is evidence that inhibition deficits contribute to the impaired word production of children with developmental language disorders, such as specific language impairment (e.g., Henry, Messer, \& Nash, 2012; Im-Bolter, Johnson, \& Pascual-Leone, 2006; SeigerGardner \& Schwartz, 2008; Spaulding, 2010). In sum, there is some evidence that inhibition may contribute to the efficiency of word production.

It has been proposed that inhibition is not a unitary construct but can best be thought of as a set of closely related abilities (e.g., Castner et al., 2007; Friedman \& Miyake, 2004; Krämer, Knight, \& Münte, 2011; Nigg, 2000; Spaulding, 2010). In the literature, several taxonomies of types of inhibition have been proposed (e.g., Friedman \& Miyake, 2004; Nigg, 2000). An important distinction is between top-down inhibitory control (e.g., Green, 1998; Jackson et al., 2001; Roelofs et al., 2011) and lateral inhibition within word planning levels (e.g., Berg \& Schade, 1992; Harley, 1993; for an extensive discussion, see Aron, 2007). The present work concerns top-down inhibitory control and, specifically, the distinction made by Forstmann et al. (2008) between nonselective and selective inhibition. Nonselective inhibition involves the top-down suppression of the planning and execution of any unwanted response. This type of inhibition is assumed to be involved in the stop-signal task, where participants prepare for a response but, upon presentation of a stop signal on a minority of trials, must refrain from executing it (e.g., Logan \& Cowan, 1984). Inhibition is taken to be nonselective because evidence suggests that the planning of any unwanted response is suppressed (e.g., Nigg, 2000). Selective inhibition involves the top-down suppression of specific strong competitors to a response, which are induced by external distractors. This type of inhibition is assumed to be involved in Stroop, Simon, and Eriksen flanker tasks (e.g., Nigg, 2000). The inhibition is taken to be selective because evidence suggests that it is specifically applied to strongly competing responses, such as the responses activated in the incongruent, but not in the congruent, condition of these tasks. Evidence from studies using Simon and Eriksen flanker tasks suggests that selective inhibition takes time to build up and, as Ridderinkhof and colleagues (Ridderinkhof, 2002; Ridderinkhof, Scheres, Oosterlaan, \& Sergeant, 2005) have shown, therefore has a stronger effect on slower than on faster responses.

Pennington (1997) found that performance on the Stroop task and the stop-signal task did not highly correlate, which suggests a distinction between selective and nonselective inhibition. This distinction is also supported by brain-imaging studies (e.g., Castner et al., 2007). For example, Krämer et al. (2011) found different ERP components as correlates for selective and nonselective inhibition. Similarly, Verbruggen, Liefooghe, and Vandierendonck (2004) obtained behavioral evidence for a difference between these two types of inhibition. However, Miyake et al. (2000) used Stroop, antisaccade, and stop-signal tasks in a latent variable analysis to explore executive functions and found a common underlying inhibition function for these three tasks (see also Friedman \& Miyake, 2004). Moreover, on the basis of findings from brain-imaging studies, other researchers have argued that selective and nonselective inhibition share a common neural locus in the right inferior frontal cortex (see Forstmann et al., 2008; Van den Wildenberg et al., 2011). In sum, the evidence on whether or not a differentiation between selective and nonselective inhibition is warranted is inconsistent.

How might the distinction between selective and nonselective inhibition apply to word production? Much of the work on the role of inhibition in word production has concerned bilingual speakers. A common assumption is that bilingual speakers use inhibition to suppress words in the nontarget language, either obligatorily (Abutalebi \& Green, 2007; Costa, Hernández, \& Sebastián-Gallés, 2008; Green, 1998; Guo et al., 2011; Jackson et al., 2001; Misra et al., 2012) or optionally (Roelofs et al., 2011; Verhoef, Roelofs, \& Chwilla, 2009). Because of the routine engagement of inhibition in language control, bilingual speakers might outperform monolingual speakers in linguistic as well as nonlinguistic tasks involving inhibitory control. This prediction has been borne out in studies using the Simon and Eriksen flanker tasks engaging selective inhibition (Bialystok, Craik, Klein, \& Viswanathan, 2004; Costa et al., 2008), but it has so far not been confirmed for other tasks requiring inhibition (Colzato et al., 2008). This suggests that bilingual speakers might primarily recruit selective inhibition in language control. However, a literature review by Hilchey and Klein (2011) revealed that many studies found no bilingual advantage in selective inhibition. A more robust finding is that bilingual individuals outperform monolingual speakers on both congruent and incongruent trials of Simon and flanker tasks, which suggests a bilingual advantage in nonselective, rather than selective, inhibition.

More central to the present research are studies of monolingual word production. Here, top-down inhibition has been invoked to explain how speakers suppress unwanted information and minimize disfluencies (Engelhardt, Corley, Nigg, \& Fereirra, 2010) or select a response among a set of competitors (de Zubicaray et al., 2002; de Zubicaray et al., 2001). Several studies used interference paradigms, where participants had to name target pictures in the presence of distractor words that were semantically or phonologically related or unrelated to the target (e.g., Glaser \& Düngelhoff, 1984; Roelofs, 1992, 2003; Schriefers et al., 1990). Given that, in these tasks, speakers have to prevent responses corresponding to highly salient competitors, selective inhibition may be involved. To the best of our knowledge, there is so far only one study, by Shao, Roelofs, and Meyer (2012), that explicitly addressed the role of nonselective inhibition in picture naming. In that study, we showed that individual differences in picture naming speed were related 
to the speakers' nonselective inhibition ability as measured through their performance in the stop-signal task.

Taken together, the available results suggest that both selective and nonselective inhibition may play a role in monolingual naming. However, since each of the existing studies assessed only one type of inhibition, nothing can be said about the relationship between the two types of inhibition. The aim of the present study was to examine this relationship by assessing both types of inhibition in the same group of participants. We used an individual-differences approach (see also Kramer, Humphrey, Larish, Logan, \& Strayer, 1994; Ridderinkhof, Band, \& Logan, 1999) and examined whether participants with good, or poor, nonselective inhibition would also show good, or poor, selective inhibition, and we examined how the individuals' speed of lexical access was affected by both types of inhibition.

The participants were tested in two tasks. One task was the stop-signal task, introduced by Logan and Cowan (1984). Here, the participants were instructed to perform a dual-choice response task. Occasionally, a stop signal was presented to indicate that participants should stop any response. The timing of the stop signal varied across trials, depending on the participant's performance on the preceding trial (see below for details). The second task was a picture-word interference task, where the participants named pictures accompanied by written distractor words that belonged to the same semantic category as the target or to a different category (e.g., target: dog, related distractor: cat, unrelated distractor: tree). A standard finding in picture-word interference experiments is that the RT is longer in the presence of same-category, as compared with unrelated, distractors (e.g., Glaser \& Düngelhoff, 1984; Glaser \& Glaser, 1989; Lupker, 1979; Lupker \& Katz, 1981). The origin of this semantic interference effect is currently under debate. One account is that it arises during lemma selection (e.g., Levelt et al., 1999; Roelofs, 1992; Schriefers et al., 1990): A semantically related distractor receives activation from the target and is, therefore, a more potent competitor to the target than an unrelated distractor, which is not activated by the target (see Roelofs, 1992, 2003, for details). An alternative account is that the semantic interference effect occurs because the articulatory program derived for the written distractor enters an articulatory buffer and must be removed for an overt response to the picture to occur. This process of removing the articulatory program for the distractor from the buffer is assumed to take longer when the distractor is semantically related to the target than when it is unrelated (e.g., Finkbeiner \& Caramazza, 2006; Mahon, Costa, Peterson, Vargas, \& Caramazza, 2007).

On both accounts of the semantic interference effect, speakers may inhibit their response to the distractor, more so for semantically related than for unrelated distractors. Their ability to do this (i.e., their selective inhibition ability) can be represented in a delta plot, which represents the size of the interference effect as a function of relative naming RT (De Jong, Liang, \& Lauber, 1994; Ridderinkhof, 2002). To compute a delta plot, the cumulative distribution of RTs for each condition is divided into quantiles (e.g., $20 \%$ bins), and the interference effect (delta) for each quantile is plotted (see Fig. 1). As Ridderinkhof has shown, in the absence of inhibition, delta increases across the quantiles: Slower reactions are accompanied by larger effects. However, when selective inhibition is applied, this increase in effect size is counteracted. As inhibition requires time to build up, this leads to a decrease of the deltas and of the slopes of the delta plot across quantiles (for reviews, see Proctor, Miles, \& Baroni, 2011; Van den Wildenberg et al., 2011). The slope of the slowest segment (e.g., the segment connecting the fourth and fifth quintiles; q4-5 in Fig. 1) appears to be most sensitive to selective inhibition ability (Forstmann et al., 2008). Therefore, this slope can be used to estimate a person's selective inhibition ability. As is shown in Fig. 1 (right panel), strong inhibition of responses to semantically related distractors may even turn semantic interference into semantic facilitation (i.e., the delta of q5 and the slope of segment q4-5 have negative values).

We expected to replicate the semantic interference effect seen in earlier studies. We assessed the correlation between the magnitude of the semantic interference effect and the slope of the slowest delta segment across participants. On the basis of the results obtained by Roelofs and colleagues (2011), we expected that the larger the magnitude of the semantic interference effect, the steeper the slope of the slowest delta segment would be (see Fig. 1). Such a relationship would indicate that the participants with smaller interference effects apply selective inhibition more effectively than do participants with larger interference effects (for extensive discussions, see Proctor et al., 2011; Van den Wildenberg et al., 2011). On the basis of the results obtained by Shao and colleagues (2012), we expected that the participants' mean RT would be correlated with their stop-signal RT. This would indicate that good nonselective inhibition (i.e., inhibition of responses to both semantically related and unrelated distractors) contributes to fast reactions in the picture naming task. The most important question concerned the relationship between nonselective inhibition (indexed by the stop-signal RT) and selective inhibition (indexed by the slope of the slowest delta segment). If they reflect the same underlying inhibition ability, as suggested by Friedman and Miyake (2004), Forstmann et al. (2008), Miyake et al. (2000), Nigg (2000), and Van den Wildenberg et al. (2011), a positive correlation should be found between the stop-signal RT and the slope of the slowest delta segment across participants. By contrast, the absence of such a correlation would suggest that nonselective and selective inhibition are separable to some extent (e.g., Castner et al., 2007; Krämer et al., 2011; Pennington, 1997; Verbruggen et al., 2004). 


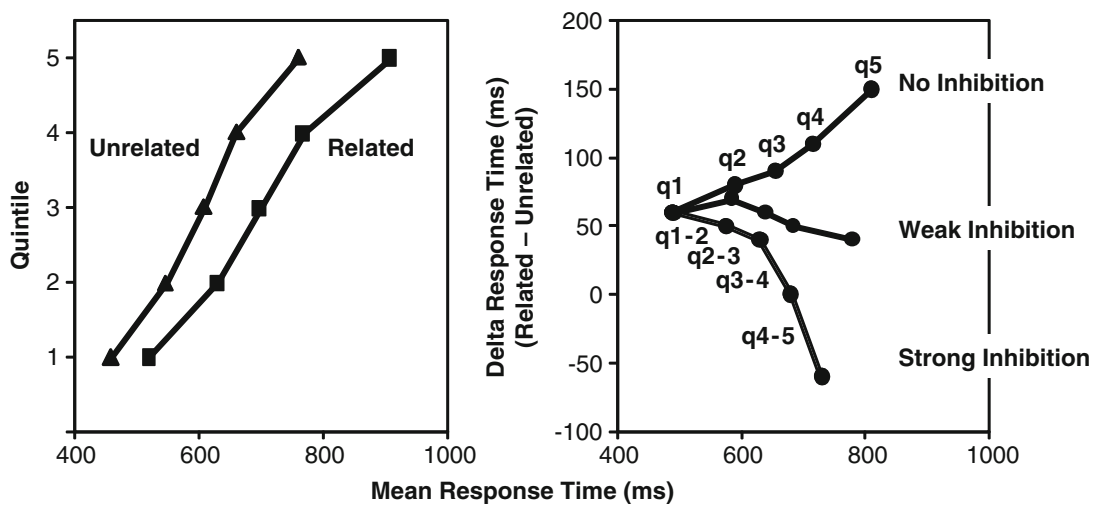

Fig. 1 Left panel: cumulative distribution curves for response times in semantically related and unrelated conditions. Right panel: delta plot showing the condition differences (deltas) as a function of quintile

\section{Method}

\section{Participants}

The study was carried out with 88 native Dutch speakers (14 men; mean age $=30.15$ years; range, $16-63$ years $^{1}$ ), selected from the participant pool of the MPI for Psycholinguistics. They participated in exchange for payment. All participants had normal or corrected-to-normal vision and normal hearing.

Picture-word interference task

\section{Materials and design}

The materials consisted of 56 line drawings of common objects adopted from the Snodgrass and Vanderwart (1980) corpus. The picture names were monosyllabic or disyllabic; the average log word-form frequency in the CELEX database was 1.25 /million $(S D=0.59)$, and the average age of acquisition was 6.76 years $(S D=1.54$ years; Ruts et al., 2004). The pictures fitted into a virtual frame of $4 \times 4 \mathrm{~cm}$ on the computer screen $\left(2.29^{\circ}\right.$ of visual angle) and were shown on a white background in the center of the computer screen.

The pictures were combined with semantically related and unrelated distractor words. Most previous work using delta-plot analyses to examine selective inhibition used Simon or flanker tasks with incongruent and congruent conditions rather than conditions with distractors being present or absent. With distractors being present or absent, it is impossible to tell whether inhibition is selective or nonselective. That is, suppression could involve selective inhibition (i.e., only the response to the distractor is inhibited) or nonselective inhibition (i.e., any incorrect response, including that to the distractor, is inhibited).

\footnotetext{
${ }^{1}$ The study was carried out in the Psychology of Language Department at the Max Planck Institute for Psycholinguistics, where a systematic effort is made to involve participants of all ages and with diverse backgrounds in the research.
}

(1-5) and amount of inhibition (no, weak, strong). q1, quintile 1, and so forth; q1-2 is the segment connecting quintiles 1 and 2, and so forth. (Adapted from Roelofs et al., 2011)

By using semantically related and unrelated distractors, it may be assessed whether inhibition is indeed selective (i.e., applied more strongly to semantically related than to unrelated distractors) or nonselective (i.e., applied equally strongly to the semantically related and unrelated distractors).

In the semantically related condition, the pictures were combined with written distractor words from the same semantic category. Targets and distractors were unrelated in phonological form; that is, they did not share the onset consonant(s) or rhyme. In the unrelated condition, the same pictures and distractor words were used, but they were recombined into semantically and phonologically unrelated pairs (see the Appendix). Figure 2 shows two example stimuli. Each picture was also shown with two further semantically unrelated distractors, one of which was phonologically related to the picture. The effects of these distractors did not differ from each other, and the corresponding trials are treated as filler trials here. The distractors were superimposed in the center of the pictures and were presented in black, in lower case Arial font of 26-point size.

Fifty-six target pictures were combined with either semantically related or unrelated distractors, which led to a total of 112 items. These 112 items were evenly distributed across four blocks, such that each block contained 28 target items. In each block, each target picture was shown only once. In addition, 28 filler items were inserted into each block. The order of the trials within each block was pseudorandomized, such that no more than three target pictures of the same condition appeared in succession, and consecutive pictures were not semantically or phonologically related. The order of the four blocks was rotated across participants.

\section{Procedure}

The participants were tested individually. At the beginning of the study, they were given a booklet showing the pictures and their names. They were first asked to familiarize themselves with the materials and to use only the names in the 


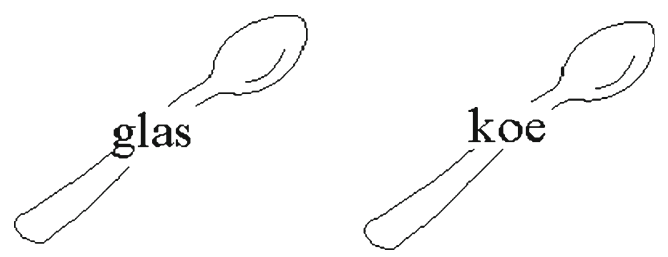

Fig. 2 Example stimuli for the semantically related (left) and unrelated (right) conditions (target: lepel [spoon]; distractors: glas [glass], koe [cow])

booklet to refer to the pictures. Then they were handed a second booklet showing only the pictures and were asked to name them. Errors were immediately corrected by the experimenter. This familiarization phase was followed by the four test blocks, which were separated by short breaks. Participants were instructed to name the pictures aloud as quickly and as accurately as possible.

On each trial of the test blocks, a fixation cross $(+)$ was presented for $300 \mathrm{~ms}$ in the center of the screen. After a blank interval of $200 \mathrm{~ms}$, a target-distractor compound was shown until the participant overtly responded, for a maximum of $3 \mathrm{~s}$. The intertrial interval was $500 \mathrm{~ms}$.

\section{Apparatus}

An HP 8540P laptop with the software package Presentation ${ }^{\circledR}$ (Version 14.3, www.neurobs.com) was used to control the experiment. Naming RTs were recorded online using a voice key but were later checked and, where necessary, corrected using the speech analyses program Praat (Boersma, 2001).

\section{Data analyses}

Responses were categorized as errors when speakers used object names that were different from those given in the picture booklet or when the response included a repair or disfluency or started with a filler word (e.g., "uh"). Errors were excluded from the RT analyses. To generate the delta plots, the RTs for each participant and distractor condition were sorted in ascending order and divided into RT quintiles (i.e., $20 \%$ bins). Then the mean RT and the average semantic effect for each condition and quintile were calculated. Following De Jong et al. (1994; see also Ridderinkhof, 2002), the slopes of the lines connecting the delta values for successive quintiles $x$ and $y$ were computed as follows:

Slope $(x, y)=\frac{\operatorname{Delta}(\text { Quintile } y)-\operatorname{Delta}(\text { Quintile } x)}{\text { Mean }(\text { Quintile } y)-\operatorname{Mean}(\text { Quintile } x)}$.

Stop-signal task

\section{Materials, design, and procedure}

The visual stimuli in the stop-signal task were a fixation cross, a square $(1.5 \times 1.5 \mathrm{~cm})$, and a circle $(1.5 \mathrm{~cm}$ in diameter). The auditory stimulus was a $750-\mathrm{Hz}$ tone with a duration of $75 \mathrm{~ms}$.

On go trials, the fixation cross $(+)$ was presented in the middle of the screen for $250 \mathrm{~ms}$ and was immediately replaced by a square or a circle for a maximum of $1,250 \mathrm{~ms}$. Squares and circles were presented equally often in a random order. The participants should press the "/" key when they saw a circle and the " $Z$ " key when they saw a square. They were instructed to respond as quickly as possible. The keypress terminated the trial. On stop-trials, the tone was played as a stop signal shortly after the offset of the fixation cross. The participants were instructed to withhold their response when they heard the tone. Initially, the stopsignal delay (SSD) was set to $250 \mathrm{~ms}$ after the offset of the fixation cross. If the participant successfully inhibited the response on a given stop trial, the delay in the following stop trial was increased by $50 \mathrm{~ms}$ (making it harder to withhold the response), otherwise the delay was decreased by $50 \mathrm{~ms}$.

There was a practice block of 32 trials, followed by three test blocks of 64 trials each. Each block included $75 \%$ go trials and $25 \%$ stop-trials, presented in a random order. Following Verbruggen, Logan, and Stevens (2008), each participant's stop-signal RT was estimated by subtracting the mean SSD from the mean RT on go trials.

\section{Apparatus}

The same laptop and experimental software were used as for the picture-word interference experiment. Sennheiser HD 201 headphones were used to present the tone.

\section{Results}

The results obtained from 4 participants were excluded from the analysis because they failed to follow the instructions in the stop-signal task. For the remaining participants, the error rate on go trials was $4.3 \%$, the RT on go trials was $645 \mathrm{~ms}$, and the estimated stop-signal RT (SSRT) was $277 \mathrm{~ms}$. The participants successfully withheld their response on $48 \%$ of the no-go trials. These values are similar to those found in earlier studies (e.g., Logan, Schachar, \& Tannock, 1997; Shao et al., 2012).

Table 1 shows the average error rates and RTs in the semantically related and unrelated conditions of the picture-word interference experiment. As was expected, the participants' responses were slower, by $39 \mathrm{~ms}$, in the related than in the unrelated condition. This semantic interference effect was significant in analyses of variance (ANOVAs) using participants $\left(t_{1}\right)$ and items $\left(t_{2}\right)$ as random variables, $t_{1}(83)=7.76, p<.001$, $t_{2}(55)=4.99, p<.001$. More errors were made in the semantically related than in the unrelated condition, but this difference was statistically not reliable, $t_{1}(83)=1.81, p=.08, t_{2}(55)=$ $1.08, p=.29$. To assess whether the semantic interference effect 
varied with test block, we submitted the RTs to a 4 (block: 1, 2, 3, 4) $\times 2$ (distractor condition: semantically related, unrelated) repeated measures ANOVA. When using participants as a random variable, there was neither a significant main effect of block, $F_{1}(3,80)=0.27, p=.85$, nor an interaction between block and distractor condition, $F_{1}(3,80)=0.53, p=.66$. When using items as random variables, there was a significant main effect of block, $F_{2}(3,168)=3.63, p<.05$, but no significant interaction between block and distractor condition, $F_{2}(3,168)=0.79, p=.50$.

The average naming RT correlated positively with the stop-signal RT, $r=.38, p<.001$. Since the average naming RT was based on the naming RT in the related and unrelated conditions, this correlation may be affected by the semantic interference effect. Therefore, we also correlated the naming RT in the unrelated condition only with the stop-signal RT and found a similar correlation, $r=.37, p<.001$.

By contrast, there was no correlation between the stop-signal RT (indexing nonselective inhibition) and the slope of slowest delta segment (indexing selective inhibition), $r=-.05, p=.62$. In line with this finding, the magnitude of the semantic interference effect and the mean stop-signal RT were not correlated either, $r=.07, p=.56$. However, the magnitude of the semantic interference effect and the slope of the slowest delta segment were correlated, $r=.55, p<.001$. Similarly, the magnitude of the semantic effect and the delta of the fifth quintile (i.e., the delta corresponding to $\mathrm{q} 5$ in Fig. 1) were correlated, $r=.50$, $p<.05$. Figure 3 shows the scatterplots for these correlations.

In computing the delta plots, we sorted the picture naming RTs for each participant in ascending order, separately for each distractor condition. Quintiles were then defined separately for each distractor condition, and the magnitude of the semantic effect was computed by subtracting the quintile average in the unrelated condition from the corresponding average in the related conditions. Therefore, a participant's responses to a given target picture in the related and unrelated conditions were not always in the same quintile. A strength of the design of the picture-word experiment is that the same target pictures are used in the related and unrelated conditions. However, this matching of pictures is lost when the items are assigned to quantiles according to the participant's RT. To address this problem, we also computed item-based delta plots, by sorting in ascending order the RTs for each item (instead of participant), for each distractor condition separately (cf. Roelofs, 2008). The quintiles, which now featured the

Table 1 Results for the picture-word interference task: mean naming response time (RT, in milliseconds) and error rate per distractor condition $(S D=$ standard deviation $)$

\begin{tabular}{lllc}
\hline Distractor Condition & Mean RT & $S D$ & Error Rate (\%) \\
\hline Related & 895 & 107 & 4.3 \\
Unrelated & 856 & 96 & 4.0 \\
\hline
\end{tabular}

same target pictures, were again defined separately for each distractor condition. The magnitude of the semantic interference effect and the slope of slowest delta segment were correlated, $r=.65, p<.001$, replicating the results of the participant-based delta plot analysis.

The strength of a correlation is constrained by the reliability of the measurements (e.g., Spearman, 1904, 1927). If reliability is not perfect, the observed correlation will be attenuated. To estimate the reliability of the stop-signal RT, we grouped the odd and even trials into separate sets, calculated the SSRT for each set, and computed the correlation between sets. This yielded a reliability estimate for the stop-signal RT of $r=.43$. To estimate the reliability of the size of the semantic interference effect, we computed the semantic effect size for each target picture and created two sets of targets, pairwise matched for effect size across the entire group of participants. We then computed the correlation across participants between the sizes of the semantic interference effect seen in the two sets of pictures. This yielded a reliability estimate of $r=.87$. To estimate the reliability of the slope of the slowest delta segment, we grouped the odd and even trials into separate sets, calculated each participant's slope of the slowest delta segment for each set of trials, and computed the correlations between sets. This yielded a reliability estimate of $r=.42$. Finally, to estimate the reliability of the naming RT, we grouped the odd and even trials into separate sets, calculated each participant's naming RT for each set of trials, and computed the correlation between sets. This yielded a reliability estimate of $r=.91$. Next, we corrected the observed correlations $r(\mathrm{x}, \mathrm{y})$ for attenuation [i.e., the reliabilities of the measurements, $r(\mathrm{x}, \mathrm{x})$ and $r(\mathrm{y}, \mathrm{y})]$ by using the formula Corrected $r(\mathrm{x}, \mathrm{y})=r(\mathrm{x}, \mathrm{y}) / \sqrt{ }(r(\mathrm{x}, \mathrm{x}) r(\mathrm{y}, \mathrm{y}))$, following Spearman (1904, 1927) and others (cf. Kline, 2000). Even after correcting for attenuation, the correlation between stop-signal RT and the slope of the slowest segment remained nonsignificant (corrected $r=-.16, p=.15$ ), and the same held for the correlation between the magnitude of the semantic effect and the stop-signal RT (corrected $r=.08, p=.47$ ).

The magnitude of the semantic interference effect, the slope of the slowest delta segment, and the SSRT all concern difference scores of measurements, for which the reliability will be lower than for the mean naming RT. Still, we found that certain difference scores correlated (i.e., the magnitude of the semantic effect and the slope of the slowest segment), whereas other difference scores did not correlate (i.e., the slope of the slowest delta segment and the SSRT), even after the corrections for attenuation. Moreover, the SSRT correlated with the naming RT, but not with the magnitude of the semantic interference effect, even though the reliability of the naming RT and magnitude of the semantic effect was comparable. This suggests that the pattern of correlations is not driven by the reliability of the measurements. 

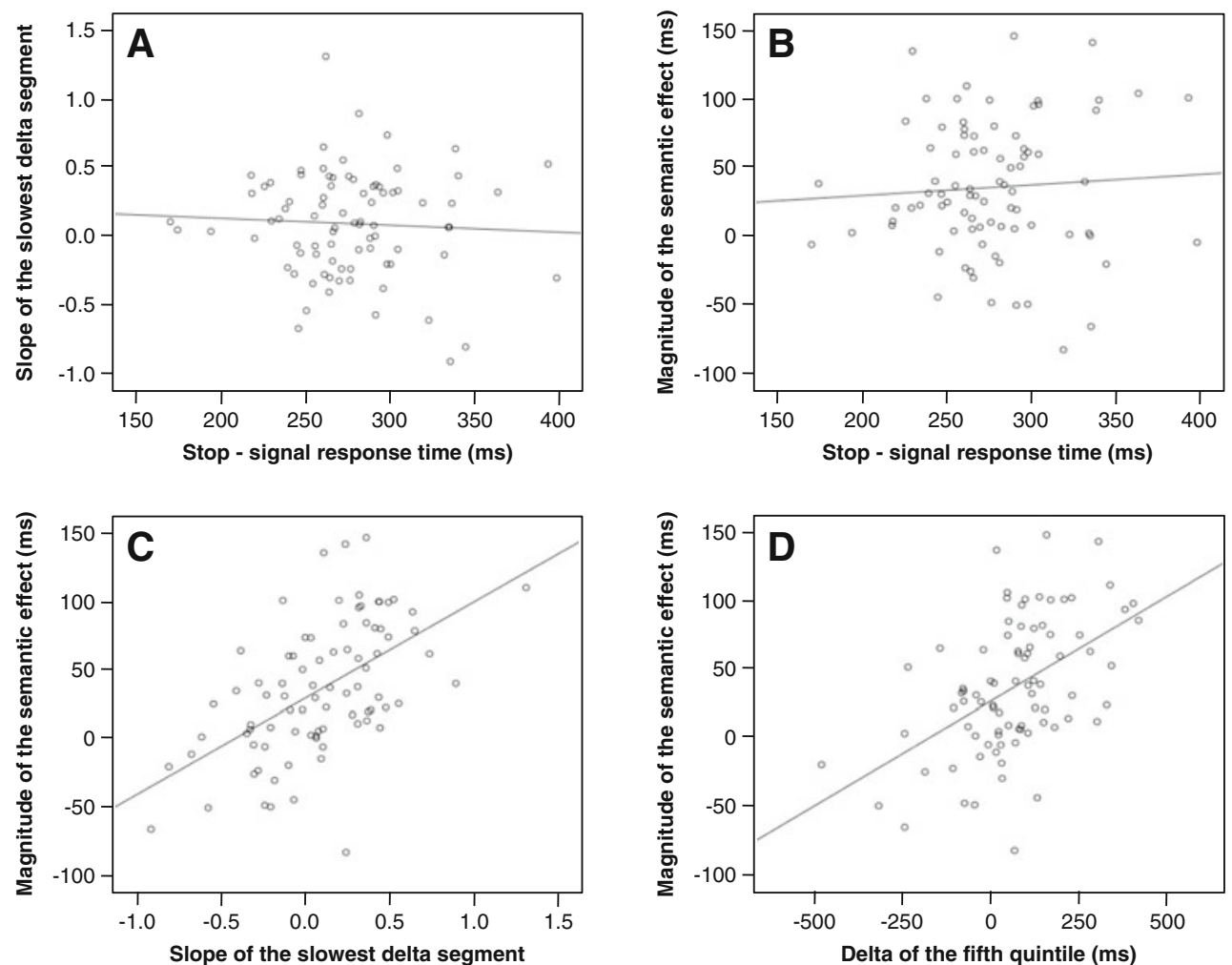

Fig. 3 Scatterplots of the relationship between a the slope of the slowest delta segment and the stop-signal response time, $\mathbf{b}$ the magnitude of the semantic interference effect and the stop-signal response

time, $\mathbf{c}$ the magnitude of the semantic interference effect and the slope of the slowest delta segment, and $\mathbf{d}$ the magnitude of the semantic interference effect and the delta of the fifth quintile

The results of the correlation analyses indicate that the slope of the slowest delta segment (indexing selective inhibition ability) and the stop-signal RT (indexing nonselective inhibition ability) are not correlated. Moreover, the magnitude of the semantic effect (depending on selective inhibition) is correlated with the slope of the slowest delta segment, but not the stop-signal RT. This pattern of results was further assessed by conducting multiple regression analyses with the magnitude of the semantic interference effect as the criterion variable and the slope of the slowest delta segment, the SSRT, and the mean naming RT as predictor variables. Table 2 shows the results. The slope of slowest delta segment (indexing selective inhibition ability) was the most significant predictor of the magnitude of semantic interference effect, $R^{2}=.38, F(3,80)=$ $16.14, p<.001$. Naming RT also made a significant contribution, but stop-signal RT (indexing nonselective inhibition ability) made no significant contribution. ${ }^{2}$

More important, we directly compared the correlations by Fischer's $r$-to- $z$ transformation test. We found that the nonsignificant correlation between the magnitude of the semantic

\footnotetext{
${ }^{2}$ We calculated Cook's distance to examine whether the regression results were driven by outliers. The values of Cook's distance ranged from 0 to .09 , mean $=.01$, and standard deviation $=.02$. Since all values were relatively low - that is, less than 1 - the results of our study were reliable trends.
}

effect and SSRT and the nonsignificant correlation between the slope of the slowest delta segment and SSRT were significantly different from the significant correlation between the magnitude of the semantic effect and the slope of the slowest delta segment, $z=-2.47, p<.01$, and $z=-3.05$, $p<.01$, respectively. The differences in correlations suggest that the contributions of selective and nonselective inhibition to picture naming are, to some extent, separable.

In the present study, we tested a sample of participants who were quite heterogeneous in terms of age and level of education. Detailed analyses of the data did not reveal any systematic moderating effects of these variables. Although age was related to SSRT, $r=.34, p<.01$, and naming RT, $r=.27, p<.05$, the correlation patterns remained the same after controlling for age. Specifically, when controlling for age, SSRT was still significantly correlated with naming RT, $r=.32, p<.01$, and the magnitude of the semantic interference effect was significantly correlated with the slope of the slowest delta segment, $r=.58$, $p<.001$. Importantly, when controlling for age, there were still no correlations between SSRT and the magnitude of the semantic interference effect, $r=.02, p=.83$, or between SSRT and the slope of the slowest delta segment, $r=.03, p=.82$.

However, the sample was not large, and therefore, subtle effects of age or education may have remained undetected. To address this concern, we carried out additional analyses 
Table 2 Results of the multiple regression analysis with the magnitude of semantic interference as criterion variable and the slope of the slowest delta segment, the stop-signal response time (SSRT), and the mean naming response time (RT) as predictor variables

\begin{tabular}{lrrl}
\hline Predictor Variables & Beta & \multicolumn{1}{l}{$S E$} & $t$-value \\
\hline Slope of slowest delta segment & 70.00 & 11.27 & $6.22^{* *}$ \\
SSRT & -.01 & .11 & -.09 \\
Mean naming RT & .13 & .05 & $2.83^{* *}$
\end{tabular}

$* * p<.01$

including only the data from young university students $(N=55$, mean age $=20.57$ years, standard deviation of age $=2.84)$. The correlation pattern for the students was similar to that obtained for all participants. In particular, we found SSRT to be correlated with mean naming RT, $r=.28, p<.05$, but not with the slope of the slowest delta segment, $r=-.07, p=.32$, or with the magnitude of the semantic interference effect, $r=.00, p=.49$. Thus, the correlation pattern for the young students was similar to that obtained for all participants.

\section{Discussion}

The ability to inhibit responses is often crucial for goaldirected, contextually appropriate behavior. Consequently, inhibitory control is widely regarded as a key component of executive control. However, it is far from clear how inhibitory control should be defined, whether it is useful to distinguish different types of inhibition and, if so, how they should be empirically distinguished, and finally, how domain-general inhibitory control processes affect specific types of behavior. In the present study, we employed a standard psycholinguistic task (picture naming in the presence of distractors) and a standard inhibition task (the stop signal task) to explore, first, how inhibition affects performance in the linguistic task and, second, whether it is useful to distinguish two types of inhibition - namely, selective and nonselective inhibition.

The study reported above yielded four key findings. First, we replicated the semantic interference effect seen in many earlier studies (e.g., Glaser \& Düngelhoff, 1984; Lupker, 1979): The participants were slower to name targets accompanied by semantically related than by unrelated distractors. As was discussed above, this semantic interference effect has been allocated at the level of lexical selection (e.g., Levelt et al., 1999) or articulatory buffering (e.g., Mahon et al., 2007). Discriminating between these accounts was not a goal of the present study.

Second, the participants differed substantially in the magnitude of the semantic effect. Delta plot analyses showed that the larger the semantic interference effect for a participant, the steeper the slope of the slowest delta segment. Since such a pattern has been shown only once before for picture-word interference in a study of bilingual naming (Roelofs et al., 2011), obtaining it in a study of monolingual naming is of importance in its own right. The finding confirms that the slope of the slowest delta segment indexed selective inhibition: Participants inhibited responses to semantically related distractors more strongly than responses to unrelated distractors.

A third finding was that the overall RT in the naming task was correlated with the stop-signal RT in the stop-signal task, replicating Shao et al. (2012). The stop-signal RT is not an indicator of absolute processing speed but a difference score indicating how quickly planned responses can be stopped. Participants with short stop-signal RTs had overall shorter naming RTs than did participants with longer stop-signal RTs.

A correlation between stop-signal RT and naming RT was observed in two earlier studies (e.g., Shao et al., 2012; Xue, Aron, \& Poldrack, 2008); the present study is the first to use the picture-word interference paradigm. In Shao et al., we found, as in the present study, that the participants' overall RT in object and action naming was positively correlated with their stopsignal RT. The correlation between stop-signal RT and naming RT was somewhat stronger than in the present study. A likely reason for this is that the picture names were harder to retrieve in the earlier study, where we used items of lower name frequency and where the participants were not familiarized with the pictures and their names before the experiment. ExGaussian analyses of the RT distributions in the earlier study demonstrated that inhibition was more consistently engaged in action naming than object naming, presumably because the action pictures were more complex and triggered more incorrect responses than did the object pictures. ${ }^{3}$ The function of nonselective inhibition is to suppress the activation of any irrelevant responses activated by the pictures. However, given that pictures presumably only activate semantically related responses, the study did not allow us to determine whether the inhibition was indeed nonselective.

Finally, we found that stop-signal RT (indexing nonselective inhibition) was not correlated with the slope of the slowest delta segment (indexing selective inhibition) or the magnitude of the semantic interference effect (depending on selective inhibition), even after correcting for attenuation (i.e., the reliability of the measurements). In evaluating this null-result, it is important to keep in mind that stop-signal RT did correlate with the overall naming RT, as just discussed. Apparently, the ability to stop any irrelevant response to a target is different from the ability to quickly suppress the response to a specific, semantically related distractor. This suggests that the type of inhibition indexed by the stop-signal RT is indeed nonselective - that is, applied equally to semantically related and unrelated competitors. Consequently, nonselective inhibition reduces general interference during

\footnotetext{
${ }^{3}$ In the present study, the number of trials per condition was too low for ex-Gaussian analyses.
} 
picture naming but has no effect on the magnitude of the semantic interference effect.

In sum, our results illustrate how a domain-general executive control process - inhibition - can affect performance in a linguistic task. They also illustrate how the effects of closely related executive control processes can be separated: We demonstrated that selective and nonselective inhibition affected the participants' naming performance in the picture-word interference task in different ways. It should, however, be noted that this conclusion is based on correlation patterns at the level of individual tasks (manifest variables), not at the level of latent variables (cf. Friedman \& Miyake, 2004). This means that the correlation patterns obtained here may be specific to the tasks we used. It is also important to point out that the indicator of selective inhibition ability was derived from the picture naming task, rather than being determined in an independent task. Conducting a latent variable analysis is out of the scope of the present study. Future studies may seek corroborating evidence for our conclusions from a large-scale study where each of the critical constructs - speed of lexical access, selective and nonselective inhibition-would be assessed in several ways so that the relationships of the underlying latent variables could be determined.

Our results imply that it is useful to distinguish between selective and nonselective inhibition. Taking account of the distinction between selective and nonselective inhibition not only is important for studies of inhibitory control per se, but also may be useful for considering the function of inhibitory control in language processes. As was mentioned in the introduction, although the role of top-down inhibition during language production processes has been increasingly noticed (de Zubicaray et al., 2002; de Zubicaray et al., 2001), the differentiation of types of inhibition has been neglected. For example, de Zubicaray and colleagues (2002; de Zubicaray et al., 2001) examined inhibition using picture naming with distractors, without distinguishing between selective and nonselective inhibition. However, the present results suggest that distractor effects only reflect selective inhibition.

Future research should consider the roles different types of inhibition may play during language processing. This should be done not only for normal adult language performance, as assessed in the present study, but also for impaired language performance. Recent research suggests that inhibition is often deficient in individuals with specific language impairment (e.g., Henry et al., 2012; Im-Bolter et al., 2006; Seiger-Gardner \& Schwartz, 2008; Spaulding, 2010). However, it is not clear which type of inhibition is affected. Specific language impairment (SLI) is a severe disorder of language acquisition and use in children who otherwise develop normally. The language disorder may persist into adulthood. The characteristics of the impaired language performance in SLI are quite variable, but common characteristics include a delay in starting to talk in childhood, deviant production of speech sounds, a restricted vocabulary, slow and inaccurate picture naming, and the use of simplified grammatical structures, including omission of articles and plural and past tense endings (see Leonard, 1998, for a review).

Seiger-Gardner and Schwartz (2008) compared the performance of children with SLI and typically developing children (on average, 9 years old) in a picture-word interference task using spoken distractor words. Stronger semantic interference was observed in the SLI than in the control group (108 vs. $43 \mathrm{~ms}$, respectively). This was taken as evidence that children with SLI were less effective in suppressing semantic alternatives. The results from the delta-plot analysis in the present study are consistent with this view. According to SeigerGardner and Schwartz, "If children with SLI have a suppression mechanism deficiency, their ability to suppress irrelevant information in non-linguistic tasks should be equally poor" (p. 546). However, the results of the present study show that this generalization from the magnitude of semantic interference to other task situations may not be warranted. In our study, the ability of selective inhibition indexed by the delta-plot analysis was not correlated with the ability of nonselective inhibition indexed by the stop-signal task. Still, picture naming RTs were generally longer for the SLI than for the typically developing group, which was attributed by Seiger-Gardner and Schwartz to general slowing. The present results-in particular, the correlation between stop-signal RT and picture-naming RT - suggest that this slowing of picture naming may reflect a difference in nonselective inhibition. In line with this interpretation of the picture naming RTs in SLI, Spaulding (2010) observed inhibition weaknesses in preschool children with SLI, as compared with typically developing controls, in a type of stop-signal task, as well as a task requiring the suppression of distractors. To conclude, evidence suggests that both selective and nonselective inhibition may be deficient in children with SLI, as compared with typically developing controls. Nevertheless, selective and nonselective inhibition may dissociate, as shown by the present study.

\section{Conclusions}

To summarize, the present study suggests separability of nonselective inhibition (as indexed by stop-signal RT) and selective inhibition (as indexed by the slope of slowest delta segment) in picture naming. The former type of inhibition is proposed to suppress any competing response, and the latter is proposed to suppress specifically alternatives that are strong competitors to a correct response. Future theoretical and empirical work on the involvement of inhibition in picture naming and, more generally, in word production should take the distinct functions of inhibition into account. 


\section{Appendix}

Table 3 Target names of pictures and semantically related and unrelated distractors, followed by English translations in parentheses

\begin{tabular}{|c|c|c|c|c|c|}
\hline Target Names & Related Distractors & Unrelated Distractors & Target Names & Related Distractors & Unrelated Distractors \\
\hline anker (anchor) & loopplank (gangway) & caravan (trailer) & kerk (church) & moskee (mosque) & schoffel (hoe) \\
\hline $\operatorname{arm}(\operatorname{arm})$ & voet (foot) & boter (butter) & ketel (kettle) & pan (frying pan) & standbeeld (statue) \\
\hline bank (couch) & dressoir (sideboard) & paleis (palace) & kok (cook) & bakker (baker) & wapen (weapon) \\
\hline beer (bear) & tijger (tiger) & mandarijn (mandarin) & kruis (cross) & driehoek (triangle) & vetplant (succulent plant) \\
\hline berg (hill) & weide (meadow) & struik (bush) & lepel (spoon) & glass (glass) & koe (cow) \\
\hline bezem (broom) & dweil (rag) & pan (frying pan) & maan (moon) & planeet (planet) & driehoek (triangle) \\
\hline bom (bomb) & mijn (mine) & vijl (file) & masker (mask) & schmink (makeup) & piano (piano) \\
\hline boom (tree) & struik (bush) & glass (glass) & motor (motorbike) & auto (car) & $\operatorname{tank}(\operatorname{tank})$ \\
\hline borstel (brush) & gel (gel) & mitrailleur (machine gun) & orgel (organ) & piano (piano) & tijger (tiger) \\
\hline bot (bone) & Spier (muscle) & helicopter (helicopter) & pijl (arrow) & speer (spear) & loopplank (gangway) \\
\hline broek (pants) & trui (sweater) & rolschaats (roller-skate) & pop (doll) & teddy beer (teddy bear) & knikker (marble) \\
\hline bus (bus) & tram $($ tram $)$ & weide (meadow) & schaar (scissors) & lijm (glue) & pijp (pipe) \\
\hline cactus (cactus) & vetplant (succulent plant) & ploeg (plow) & schip (ship) & onderzeër (submarine) & mijn (mine) \\
\hline citroen (lemon) & mandarijn (mandarin) & ventiel (valve) & sigaar (cigar) & pijp (pipe) & moskee (mosque) \\
\hline fluit (flute) & hoorn (horn) & teddy beer (teddy bear) & slak (snail) & worm (worm) & behang (wallpaper) \\
\hline fontein (fountain) & standbeeld (statue) & oor (ear) & step (scooter) & rolschaats (roller-skate) & kantoor (office) \\
\hline gordijn (curtain) & behang (wallpaper) & cello (cello) & ster (star) & meteoor (meteor) & hoorn (horn) \\
\hline hamer (hammer) & vijl (file) & dweil (rag) & tas (bag) & koffer (suitcase) & lijm (glue) \\
\hline hand (hand) & oor (ear) & marmot (marmot) & tent (tent) & caravan (trailer) & band (tire) \\
\hline harp (harp) & trompet (trumpet) & spier (muscle) & tol (top) & knikker (marble) & slinger (garland) \\
\hline hengel (fishing rod) & dobber (float) & teen (toe) & tractor (tractor) & ploeg (plow) & koffer (suitcase) \\
\hline hoed (hat) & pet (cap) & beker (mug) & vaas (vase) & pot (jar) & trompet (trumpet) \\
\hline jurk (dress) & blouse (blouse) & onderzeër (submarine) & varken (piglet) & koe (cow) & auto (car) \\
\hline kaas (cheese) & boter (butter) & rechthoek (rectangle) & vinger (finger) & teen (tor) & shampoo (shampoo) \\
\hline kam (comb) & shampoo (shampoo) & planeet (planet) & vlag (flag) & wapen (weapon) & meteoor (meteor) \\
\hline kameel (camel) & aap (ape) & bakker (baker) & vork (fork) & servet (napkin) & schmink (makeup) \\
\hline kan (can) & beker (mug) & glijbaan (slide) & wiel (wheel) & band (tire) & speer (spear) \\
\hline kanon (cannon) & $\operatorname{tank}(\operatorname{tank})$ & bed (bed) & zaag (saw) & tang (tongs) & voet (foot) \\
\hline
\end{tabular}

\section{References}

Abutalebi, J., \& Green, D. (2007). Bilingual language production: The neurocognition of language representation and control. Journal of Neurolinguistics, 20, 242-275.

Aron, A. R. (2007). The neural basis of inhibition in cognitive control. The Neuroscientist, 13, 214-228.

Baddeley, A. D. (1986). Working memory. Oxford, England: Oxford University Press.

Badecker, W., Miozzo, M., \& Zanuttini, R. (1995). The two-stage model of lexical retrieval: Evidence from a case of anomia with selective preservation of grammatical gender. Cognition, 57, 193-216.

Berg, T., \& Schade, U. (1992). The role of inhibition in a spreadingactivation model of language production. II. The simulational perspective. Journal of Psycholinguistic Research, 21, 435-462.

Bialystok, E., Craik, F. I. M., Klein, R., \& Viswanathan, M. (2004). Bilingualism, aging, and cognitive control: Evidence from the Simon task. Psychology and Aging, 19, 290-303.

Boersma, P. (2001). Praat, a system for doing phonetics by computer. Glot International, 5, 341-345.
Caramazza, A. (1997). How many levels of processing are there in lexical access? Cognitive Neuropsychology, 14, 177-208.

Castner, J. E., Copland, D. A., Coyne, T. J., Sinclair, F., Silburn, P. A., \& Chenery, H. J. (2007). Lexical-semantic inhibitory mechanisms in Parkinson's disease: Stimulation of the subthalamic nucleus modulates behavioural inhibition but not interference control. Neuropsychologia, 45, 3167-3177.

Colzato, L. S., Bajo, M. T., van den Wildenberg, W., Paolieri, D., Nieuwenhuis, S. T., La Heij, W., et al. (2008). How does bilingualism improve executive control? a comparison of active and reactive inhibition mechanisms. Journal of Experimental Psychology: Learning, Memory, and Cognition, 34, 302-312.

Costa, A., Hernández, M., \& Sebastián-Gallés, N. (2008). Bilingualism aids conflict resolution: Evidence from the ANT task. Cognition, 106, 59-86.

De Jong, R., Liang, C.-C., \& Lauber, E. (1994). Conditional and unconditional automaticity: A dual-process model of effects of spatial stimulus-response correspondence. Journal of Experimental Psychology. Human Perception and Performance, 20, 731-750.

de Zubicaray, G. I., Wilson, S. J., McMahon, K. K., \& Muthiah, S. (2001). The semantic interference effect in the picture-word 
paradigm: An event-related fMRI study employing overt responses. Human Brain Mapping, 14, 218-227.

de Zubicaray, G. I., McMahon, K. L., Eastburn, M. M., \& Wilson, S. J. (2002). Orthographic/phonological facilitation of naming responses in the picture-word task: An event-related fMRI study using overt vocal responding. NeuroImage, 16, 1984-1093.

de Zubicaray, G., McMahon, K., Eastburn, M., \& Pringle, A. (2006). Top-down influences on lexical selection during spoken word production: A 4T fMRI investigation of refractory effects in picture naming. Human Brain Mapping, 27, 864-873.

Dell, G. S. (1986). A spreading activation theory of retrieval in language production. Psychological Review, 93, 283-321.

Dell, G. S., Schwartz, M. F., Martin, N., Saffran, E. M., \& Gagnon, D. A. (1997). Lexical access in aphasic and nonaphasic speakers. Psychological Review, 104, 801-838.

Engelhardt, P. E., Corley, M., Nigg, J. T., \& Ferreira, F. (2010). The role of inhibition in the production of disfluencies. Memory \& Cognition, 38, 617-628.

Finkbeiner, M., \& Caramazza, A. (2006). Lexical selection is not a competitive process: A reply to La Heij, Kuipers and Starreveld. Cortex, 42, 1032-1035.

Forstmann, B. U., Jahfari, S., Scholte, H. S., Wolfensteller, U., van den Wildenberg, W. P. M., \& Ridderinkhof, K. R. (2008). Function and structure of the right inferior frontal cortex predict individual differences in response inhibition: A model-based approach. Journal of Neuroscience, 28, 9790-9796.

Foygel, D., \& Dell, G. S. (2000). Models of impaired lexical access in speech production. Journal of Memory and Language, 43, 182-216.

Friedman, N. P., \& Miyake, A. (2004). The relations among inhibition and interference cognitive functions: A latent variable analysis. Journal of Experimental Psychology. General, 133, 101-135.

Glaser, W. R., \& Düngelhoff, F. J. (1984). The time course of pictureword interference. Journal of Experimental Psychology. Human Perception and Performance, 10, 640-654.

Glaser, W. R., \& Glaser, M. O. (1989). Context effects in Stroop-like word and picture-processing. Journal of Experimental Psychology. General, 118, 13-42.

Goodglass, H., Kaplan, E., Weintraub, S., \& Ackerman, N. (1976). The "tip-of-the-tongue" phenomenon in aphasia. Cortex, 12, 145-153.

Green, D. W. (1998). Mental control of the bilingual lexico-semantic system. Bilingualism: Language and Cognition, 1, 67-81.

Guo, T., Liu, H., Misra, M., \& Kroll, J. F. (2011). Local and global inhibition in bilingual word production: fMRI evidence from Chinese-English bilinguals. NeuroImage, 56, 2300-2309.

Harley, T. A. (1993). Phonological activation of semantic competitors during lexical access in speech production. Language \& Cognitive Processes, 8, 291-309.

Henry, L. A., Messer, D. J., \& Nash, G. (2012). Executive functioning in children with specific language impairment. Journal of Child Psychology and Psychiatry, 53, 37-45.

Hilchey, M. D., \& Klein, R. M. (2011). Are there bilingual advantages on nonlinguistic interference tasks? implications for the plasticity of executive control processes. Psychonomic Bulletin \& Review, 18, 625-658.

Im-Bolter, N., Johnson, J., \& Pascual-Leone, J. (2006). Processing limitations in children with specific language impairment: The role of executive function. Child Development, 77, 1822-1841.

Indefrey, P., \& Levelt, W. J. M. (2004). The spatial and temporal signatures of word production components. Cognition, 92, 101-144.

Jackson, G. M., Swainson, R., Cunnington, R., \& Jackson, S. R. (2001). ERP correlates of executive control during repeated language switching. Bilingualism: Language and Cognition, 4, 169-178.

Kay, J., \& Ellis, A. W. (1987). A cognitive neuropsychological case study of anomia: Implications for psychological models of word retrieval. Brain, 110, 613-629.

Kline, P. (2000). The handbook of psychological testing. London, UK: Routledge.
Kramer, A. F., Humphrey, D. G., Larish, J. F., Logan, G. D., \& Strayer, D. L. (1994). Aging and inhibition: Beyond a unitary view of inhibitory processing in attention. Psychology and Aging, 9, 491-512.

Krämer, U. M., Knight, R. T., \& Münte, T. F. (2011). Electrophysiological evidence for different inhibitory mechanisms when stopping or changing a planned response. Journal of Cognitive Neuroscience, 23, 2481-2493.

Leonard, L. B. (1998). Children with specific language impairment. Cambridge, MA: MIT Press.

Levelt, W. J. M. (2001). Spoken word production: A theory of lexical access. Proceedings of the National Academy of Science, 98, 13464-13471.

Levelt, W. J. M., Roelofs, A., \& Meyer, A. S. (1999). A theory of lexical access in speech production. The Behavioral and Brain Sciences, 22, 1-37.

Logan, G. D., \& Cowan, W. B. (1984). On the ability to inhibit thought and action: A theory of an act of control. Psychological Review, 91, 295-327.

Logan, G. D., Schachar, R. J., \& Tannock, R. (1997). Impulsivity and inhibitory control. Psychological Science, 8, 60-64.

Lupker, S. J. (1979). The semantic nature of response competition in the picture-word interference task. Memory \& Cognition, 7, 485-495.

Lupker, S. J., \& Katz, A. N. (1981). Input, decision, and response factors in picture-word interference. Journal of Experimental Psychology: Human Learning and Memory, 7, 269-282.

Mahon, B. Z., Costa, A., Peterson, R., Vargas, K., \& Caramazza, A. (2007). Lexical selection is not by competition: A reinterpretation of semantic interference and facilitation effects in the pictureword interference paradigm. Journal of Experimental Psychology: Learning, Memory, and Cognition, 33, 503-535.

Misra, M., Guo, T., Bobb, S. C., \& Kroll, J. F. (2012). When bilinguals choose a single word to speak: Electrophysiological evidence for inhibition of the native language. Journal of Memory and Language, 67, 224-237.

Miyake, A., Friedman, N. P., Emerson, M. J., Witzki, A. H., Howerter, A., \& Wager, T. D. (2000). The unity and diversity of executive functions and their contributions to complex "frontal lobe" tasks: A latent variable analysis. Cognitive Psychology, 41, 49-100.

Nigg, J. T. (2000). On inhibition/disinhibition in developmental psychopathology: Views from cognitive and personality psychology and a working inhibition taxonomy. Psychological Bulletin, 126, 220-246.

Pennington, B. F. (1997). Dimensions of executive functions in normal and abnormal development. In N. A. Krasnegor, G. R. Lyon, \& P. S. Goldman-Rakic (Eds.), Development of the prefrontal cortex: Evolution, neurobiology, and behavior (pp. 265-281). Baltimore: P.H. Brookes.

Posner, M. I., \& Petersen, S. E. (1990). The attention system of the human brain. Annual Review of Neuroscience, 13, 25-42. doi:10.1146/annurev.ne.13.030190.000325

Proctor, R. W., Miles, J. D., \& Baroni, G. (2011). Reaction time distribution analysis of spatial correspondence effects. Psychonomic Bulletin \& Review, 18, 242-266.

Rapp, B., \& Goldrick, M. (2000). Discreteness and interactivity in spoken word production. Psychological Review, 107, 460-499.

Ridderinkhof, K. R. (2002). Activation and suppression in conflict tasks: Empirical clarification through distributional analyses. In W. Prinz \& B. Hommel (Eds.), Attention and performance XIX: Common mechanisms in perception and action (pp. 494-519). Oxford: Oxford University Press.

Ridderinkhof, K. R., Band, G. P. H., \& Logan, G. D. (1999). A study of adaptive behavior: Effects of age and irrelevant information on the ability to inhibit one's actions. Acta Psychologica, 101, 315-337.

Ridderinkhof, K. R., Scheres, A., Oosterlaan, J., \& Sergeant, J. (2005). Delta plots in the study of individual differences: New tools reveal response inhibition deficits in $\mathrm{AD} / \mathrm{HD}$ that are eliminated by methylphenidate treatment. Journal of Abnormal Psychology, $114,197-215$. 
Roelofs, A. (1992). A spreading-activation theory of lemma retrieval in speaking. Cognition, 42, 107-142.

Roelofs, A. (2003). Goal-referenced selection of verbal action: Modeling attentional control in the Stroop task. Psychological Review, 110, 88-125.

Roelofs, A. (2008). Dynamics of the attentional control of word retrieval: Analyses of response time distributions. Journal of Experimental Psychology. General, 137, 303-323.

Roelofs, A., Piai, V., \& Garrido Rodriguez, G. (2011). Attentional inhibition in bilingual naming performance: Evidence from delta-plot analyses. Frontiers in Psychology, 2, article 184.

Ruts, W., De Deyne, S., Ameel, E., Vanpaemel, W., Verbeemen, T., \& Storms, G. (2004). Flemish norm data for 13 natural concepts and 343 exemplars. Behavior Research Methods, Instruments, \& Computers, 36, 506-515.

Schriefers, H., Meyer, A. S., \& Levelt, W. J. M. (1990). Exploring the time course of lexical access in language production: Pictureword interference studies. Journal of Memory and Language, 29, 86-102.

Seiger-Gardner, L., \& Schwartz, R. G. (2008). Lexical access in children with and without specific language impairment: A cross-modal picture-word interference study. International Journal of Language \& Communication Disorders, 43, 528-551.

Shao, Z., Roelofs, A., \& Meyer, A. S. (2012). Sources of individual differences in the speed of naming objects and actions: The contribution of executive control. Quarterly Journal of Experimental Psychology, 65, 1927-1944.
Snodgrass, L., \& Vanderwart, M. (1980). A standardized set of 260 pictures: Norms for name agreement, image agreement, familiarity, and visual complexity. Journal of Experimental Psychology: Human Learning and Memory, 6, 174-215.

Spaulding, T. J. (2010). Investigating mechanisms of suppression in preschool children with specific language impairment. Journal of Speech, Language, and Hearing Research, 53, 725-738.

Spearman, C. (1904). "General intelligence", objectively determined and measured. The American Journal of Psychology, 15, 201-292.

Spearman, C. (1927). The abilities of man: Their nature and measurement. London, UK: Macmillan and co.

Van den Wildenberg, W. P. M., Wylie, S. A., Forstmann, B. U., Burle, B., Hasbroucq, T., \& Ridderinkhof, R. K. (2011). To head or to heed? beyond the surface of selective action inhibition: A review. Frontiers in Human Neuroscience, 4, 1-13.

Verbruggen, F., Liefooghe, B., \& Vandierendonck, A. (2004). The interaction between stop signal inhibition and distracter interference in the flanker and Stroop task. Acta Psychologica, 116, 21-37.

Verbruggen, F., Logan, G. D., \& Stevens, M. A. (2008). STOP-IT: Windows executable software for the stop signal paradigm. Behavior Research Methods, 40, 479-483.

Verhoef, K. M. W., Roelofs, A., \& Chwilla, D. J. (2009). Role of inhibition in language switching: Evidence from event-related brain potentials in overt picture naming. Cognition, 110, 84-99.

Xue, G., Aron, A. R., \& Poldrack, R. A. (2008). Common neural substrates for inhibition of spoken and manual responses. Cerebral Cortex, 18, 1923-1932. 